\title{
Reliability, Validity and Feasibility of the Vitiligo Extent Score (VES) and Self-Assessment Vitiligo Extent Score (SA-VES) Among Vitiligo Patients: A Cross-Cultural Validation
}

\section{Pichanee Chaweekulrat Narumol Silpa-archa Chalermkwan Apinuntham Chayada Chaiyabutr (D) Chanisada Wongpraparut (D)}

Department of Dermatology, Faculty of Medicine Siriraj Hospital, Mahidol University, Bangkok, Thailand
Correspondence: Narumol Silpa-archa Department of Dermatology, Faculty of Medicine Siriraj Hospital, Mahidol

University, 2 Wanglang Road, Bangkoknoi, Bangkok, 10700, Thailand

Tel +66 2-419-4333

Fax +66 2-4II-503I

Email doctornarumol@gmail.com
Background: The Vitiligo Extent Score (VES) and Self-Assessment Vitiligo Extent Score (SA-VES) have not been formally validated in Thai population.

Objective: To evaluate reliability, validity and feasibility of the VES and SA-VES in Thai vitiligo patients.

Methods: Vitiligo lesions from 100 patients were evaluated by 2 independent dermatologists using VES and Vitiligo Area Scoring Index (VASI). Reliability was assessed by comparing VES scores between physicians. Validity was determined by comparison among the VES, VASI, and SA-VES instruments. Patients scored their vitiligo severity using the SA-VES.

Results: The reliability of the VES was excellent (inter-rater reliability: 0.997, 95\% confidence interval: 0.995-0.998). There was very strong correlation between the VES and VASI $(r=0.976, p<0.001)$, and strong correlation between the VES and SA-VES $(r=0.890$, $p<0.001)$, and between the VASI and SA-VES $(r=0.866, p<0.001)$. Moderate correlation among the VES, VASI, and SA-VES was observed in patients with segmental or $<1 \%$ body surface area (BSA) vitiligo. Ninety-five percent of patients rated the SA-VES as easy to moderately easy.

Conclusion: The VES has reliability and validity comparable to that of the VASI. The SAVES is an user-friendly instrument that correlated well with physicians' scoring methods in patients with non-segmental or $>1 \%$ BSA vitiligo.

Keywords: reliability, validity, feasibility, Vitiligo Extent Score, VES, Self-Assessment Vitiligo Extent Score, SA-VES, Thai vitiligo patients

\section{Introduction}

Vitiligo is a common acquired depigmentation that is characterized by well-defined depigmented macules and patches that result from melanocyte destruction. ${ }^{1}$ The prevalence of vitiligo was reported to be approximately $0.5-2 \%$ of the world's population. ${ }^{2}$ The pathogenesis is multifactorial with genetics, autoimmunity, oxidative stress, and environmental factors all having been implicated. ${ }^{3}$ The severity of vitiligo varies according to the percentage of area affected, which can range from small focal depigmented lesions to generalized lesions, and the stability of the disease.

Several scoring systems are currently available for assessing the clinical severity of vitiligo. These methods range from subjective method (eg, grading repigmentation and vitiligo disease activity score), semi-subjective method (Vitiligo Area Scoring Index 
$[\mathrm{VASI}]^{4}$ and Vitiligo European Task Force $\left.[\mathrm{VETF}]^{5}\right)$, and objective method, such as digital image analysis. ${ }^{6}$ However, there is still no standardized method. The VASI, which was developed by Hamzavi et $\mathrm{al}^{4}$ is one of the most frequently cited measurement methods for assessing vitiligo. In brief, the VASI score is derived by summation of the percentage of vitiligo involvement at six body regions multiplied by residual depigmentation. The depigmentation degree was validated as $100 \%, 90 \%, 75 \%, 50 \%, 25 \%$, and $10 \%{ }^{4}$ The percentage of vitiligo involvement is calculated using the Palmar method. One hand, including fingers, is equal to $1 \%$ of the body surface area (BSA). However, VASI is limited by inter-individual variation relative to determination of the amount of pigmentation and area of vitiligo involvement. ${ }^{6}$

The Vitiligo Extent Score (VES) is another clinical scoring instrument that was proposed by van Geel et al and an international Vitiligo Score Working Group in $2016 .^{7}$ The VES is a template of vitiligo images that measures vitiligo at 19 different areas of the body. However, the palms of the hands, the soles of the feet, and the back of the scalp are not included in the measurement. The severity of vitiligo in the template images range from no lesion to almost 100\% lesion coverage. The physician has to score these 19 body areas separately by selecting the image that most resembles to patient's clinical in that body area. There are + and - signs that can be selected if there is slightly more or less vitiligo lesion or use $1 / 2$ and $1 / 4$ in the first picture column. In the last column, $>75 \%$ or $100 \%$ (to reflect total depigmentation) can be selected. Total VES is calculated from the converting table by summing up the measurements from all locations by accessing www.vitiligo-calculator.com. ${ }^{7,8}$ Previous validation study showed the VES to be superior to the VASI in terms of reliability and user-friendliness. ${ }^{4,7}$

An international Vitiligo Score Working Group developed the Self-Assessment Vitiligo Extent Score (SA-VES) in 2017. ${ }^{8}$ The SA-VES is a patient-reported outcome measurement instrument that is similar to the VES, but it is easier and less complicated to complete. It includes only 12 body areas with none of the subscoring options. Since the SA-VES employs the use of only images, it can be used by vitiligo patients that speak any language. The validation of the SA-VES revealed excellent reliability and correlation with the VES. Most patients found the SAVES easy to use, and it required only a short time to complete for most patients. ${ }^{8}$ However, the majority of patients enrolled in the validation studies for the VES and the SA-VES had skin type II or III, which does not reflect the skin type of much of the world's population. In this study, we evaluate the reliability, validity and feasibility of the VES and SA-VES in Asian population, specificity in Thai vitiligo patients.

\section{Materials and Methods}

According to the intraclass correlation coefficient (ICC) of VES and SA-VES in the previous study, the calculated sample size of this study were 97 patients. ${ }^{7,8}$ This validation study included 100 vitiligo patients who attended the Department of Dermatology, Faculty of Medicine Siriraj Hospital, Mahidol University, Bangkok, Thailand, during the December 2019 to November 2020 period. We enrolled patients aged at least 18 years who were clinically diagnosed with any type of vitiligo. We included vitiligo patients who were and who were not currently receiving treatment for the disease. Patients with communication deficits or with coexisting dermatological and/or mental illness were excluded. The protocol for this study was approved by the Siriraj Institutional Review Board (SIRB) (approval no. Si 512/2019). All included patients provided written informed consent to participate.

The validation process included patient scoring method (SA-VES), and physician scoring methods (VES and VASI). Patients were given a short verbal instruction in how to complete the online SA-VES form, and then were asked to score their vitiligo severity using this form. The time needed to complete the form was recorded for purposes of evaluating the feasibility. After scoring, the patients were asked to record the difficulty of completing the SA-VES online form on a 5-point scale, as follows: very easy, easy, moderately easy, difficult, and very difficult. Afterwards, patients' vitiligo lesions were then evaluated and scored by 2 independent dermatologists (PC and NS). Standardized scoring sheets were used for scoring the VASI, whereas scoring of the VES and SA-VES was performed online at www.vitiligo-calculator.com. ${ }^{7,8}$

For reliability, we assessed inter-rater reliability (IRR) by comparing both the VES score and the VASI score between the two physicians. Validity was determined by comparing between the results of physician scoring for both the VES and the VASI, and by comparing physician scoring to patient scoring (VES and SA-VES, VASI and SA-VES). For both reliability and validity, we performed subgroup analysis specific to percent body surface area (BSA) of vitiligo lesions, type of vitiligo, and disease status. BSA involvement was categorized into 3 groups: $<1 \%, 1-9.99 \%$, and $>10 \%$. Type of vitiligo was classified 
as segmental, non-segmental, and focal vitiligo. Disease status was categorized as either active or stable disease. Active vitiligo was defined as the presence of trichrome lesions, confetti-like lesions, inflammatory vitiligo, Köebner's phenomenon, and/or new vitiligo lesions within the preceding 3 months.

\section{Statistics Analysis}

Descriptive statistics were employed to summarize patient demographics and clinical characteristics. Categorical variables are described using number and percentage, and continuous variables are shown as median and interquartile range (IQR). The inter-rater reliability (IRR) of the VES and VASI was determined by intraclass correlation coefficient (ICC). The ICC was calculated in a 2-way mixed model with absolute agreement, and was reported as single measures. ICC values of $>0.9,0.75-0.9,0.5-$ 0.75 , and $<0.5$ indicate excellent, good, moderate, and poor reliability, respectively. ${ }^{9}$ The smallest detectable change (SDC) was calculated to determine change beyond measurement error using the formula $\mathrm{SDC}_{95}=1.96 \times \sqrt{2}$ $\mathrm{x}$ Standard Error of Measurement (SEM) while, SEM = SD $\sqrt{1-\mathrm{ICC}}$. To determine validity, physician and patient scores were compared using Spearman correlation coefficients. A correlation coefficient greater than 0.9 indicates very strong correlation, while correlation coefficients of $0.7-0.89,0.4-0.69$, and $0.1-0.39$ indicate strong, moderate, and weak correlations, respectively. ${ }^{10}$ Bland-Altman plots were used to quantify the agreement between two quantitative measurements, and to detect systematic differences. Statistical analysis was performed using SPSS 22.0 (SPSS Science, Chicago, IL, USA) and MedCalc 19.6.1 (MedCalc Software Ltd, Ostend, Belgium). For all results, a two-sided $p$-value $\leq 0.05$ was considered to indicate statistical significance.

\section{Results}

Demographics and clinical characteristics of patients are shown in Table 1. Of the 100 included patients, $77 \%$ were female. Patient age ranged from 18 to 84 years, with a median age of 42 years (interquartile range [IQR]: 32.3-55.0). The majority of enrolled patients had skin type IV (59\%), followed by skin type III (25\%) and type $\mathrm{V}(16 \%)$. Concerning the type of vitiligo, $80 \%, 15 \%$, and $5 \%$ of patients had non-segmental, segmental, and focal vitiligo, respectively. Among the patients with nonsegmental type, most patients (67\%) had generalized vitiligo. More than half $(56 \%)$ of patients had lesion $<1 \%$ BSA. The median BSA percentage of overall patients based on VES score was $0.89 \%$ (IQR: 0.44-3.02). The median VASI and SA-VES scores were 0.91\% (IQR: $0.40-3.62$ ) and $0.96 \%$ (IQR: $0.41-3.21$ ), respectively.

\section{Reliability}

The ICC of overall VES and VASI were 0.997 (95\% CI: 0.995-0.998) and 0.988 (95\% CI: 0.982-0.992), respectively (Table 2). $\mathrm{SDC}_{95}$ were $2.71 \%$ for the VES and $5.18 \%$ for the VASI.

Concerning BSA percentage, all patients with vitiligo lesion $<1 \%$ BSA, 1-9.99\% BSA, and $\geq 10 \%$ BSA had good to excellent correlation for both VES and VASI score. However, $\mathrm{SDC}_{95}$ of the VASI in patients with $>10 \%$ BSA vitiligo lesion were higher than the VES. Regarding type of vitiligo, both segmental and nonsegmental type reported high ICCs for both VES and VASI score; however, the ICC of focal-type vitiligo showed only moderate reliability for VASI score. Vitiligo patients with active or stable disease also showed excellent inter-rater reliability for both VES and VASI score (Table 2).

\section{Validity}

We assessed correlation between physician (VES, VASI) and patient scores (SA-VES). As a result of the observed excellent inter-rater reliability of the VES and VASI between two physicians, we decided to use the mean values of the VES and VASI from both physicians for further analysis. The Spearman correlation coefficients showed very strong correlation between the VES and VASI ( $r=0.976, p<0.001$ ), and strong correlation between the VES and SA-VES $(r=0.890, p<0.001)$, and between the VASI and SA-VES $(r=0.866, p<0.001)$ (Figure 1). Bland-Altman analyses revealed no systematic differences between any comparisons (Figure 2).

The correlations between the VES and SA-VES at different areas of the body are shown in Table 3. The face had the lowest correlation compared to other areas $(r=0.587, p<0.001)$. Patients with higher BSA percentage of vitiligo lesions seemed to have higher correlations among the VES, VASI, and SA-VES compared to patients with lower BSA percentage. Especially in the $<1 \% \mathrm{BSA}$ group, the correlation between the VES and VASI was strong $(r=0.892, p<0.001)$, but the correlations between the VES and SA-VES, and between the VASI and SAVES were only moderate ( 0.537 and 0.584 , respectively; 
Table I Demographic and Clinical Characteristics of 100 Vitiligo Patients

\begin{tabular}{|c|c|c|c|c|}
\hline \multirow[t]{2}{*}{ Characteristics } & \multirow[t]{2}{*}{ All Vitiligo Patients } & \multicolumn{3}{|c|}{ BSA $\%$} \\
\hline & & $<1 \%$ & I-9.99\% & $\geq 10 \%$ \\
\hline Number of patients & 100 & 56 & 32 & 12 \\
\hline \multicolumn{5}{|l|}{ Gender, n (\%) } \\
\hline Male & $23(23.0 \%)$ & $13(23.2 \%)$ & $8(25.0 \%)$ & $2(16.7 \%)$ \\
\hline Female & 77 (77.0\%) & $43(76.8 \%)$ & $24(75.0 \%)$ & $10(83.3 \%)$ \\
\hline Age (yrs), median (IQR) & $42(32.3-55)$ & $39.5(31-53)$ & $39(18-73)$ & $50(45.8,63.8)$ \\
\hline \multicolumn{5}{|l|}{ Photo skin type, n (\%) } \\
\hline III & $25(25.0 \%)$ & $17(30.4 \%)$ & $6(18.8 \%)$ & $2(16.7 \%)$ \\
\hline IV & $59(59.0 \%)$ & $34(60.7 \%)$ & $18(56.3 \%)$ & 7 (58.3\%) \\
\hline $\mathrm{V}$ & $16(16.0 \%)$ & $5(8.9 \%)$ & $8(25.0 \%)$ & $3(25.0 \%)$ \\
\hline Age of onset (yrs), median (IQR) & $34.5(24.5-49.3)$ & $35(23-49)$ & $32(25-54)$ & $33.5(17.8-54)$ \\
\hline Disease duration (yrs), median (IQR) & $3(1-10)$ & $2(0.7-5.8)$ & $6.5(3-10)$ & $7(1.1-29)$ \\
\hline \multicolumn{5}{|l|}{ Type of vitiligo, n (\%) } \\
\hline Segmental & $15(15.0 \%)$ & $10(17.9 \%)$ & $4(12.5 \%)$ & I (8.3\%) \\
\hline Unisegmental & $13(13.0 \%)$ & $9(16.1 \%)$ & $3(9.4 \%)$ & I (8.3\%) \\
\hline Bisegmental & $2(2.0 \%)$ & I (I.8\%) & I (3.1\%) & $0(0.0 \%)$ \\
\hline Non-segmental & $80(80.0 \%)$ & $4 \mathrm{l}(73.2 \%)$ & $28(87.5 \%)$ & II (9I.7\%) \\
\hline Generalized & $67(67.0 \%)$ & $30(53.6 \%)$ & $26(81.3 \%)$ & II (9I.7\%) \\
\hline Acrofacial & $13(13.0 \%)$ & II (19.6\%) & $2(6.3 \%)$ & $0(0.0 \%)$ \\
\hline Unisegmental & $5(5.0 \%)$ & $5(8.9 \%)$ & $0(0.0 \%)$ & $0(0.0 \%)$ \\
\hline BSA\% (based on VES), median (IQR) & $0.89(0.44-3.02)$ & $0.48(0.25-0.74)$ & $2.49(1.49-6.88)$ & $18.29(11.89-26.13)$ \\
\hline \multicolumn{5}{|l|}{ Area of involvement, $n$ (\%) } \\
\hline Face & $53(53.0 \%)$ & $28(50.0 \%)$ & I8 (56.3\%) & $7(58.3 \%)$ \\
\hline Trunk & 49 (49.0\%) & $18(32.1 \%)$ & 21 (65.6\%) & $10(83.3 \%)$ \\
\hline Extremities & 51 (5I.0\%) & $20(35.7 \%)$ & $20(62.5 \%)$ & II (91.7\%) \\
\hline Feet & $53(53.0 \%)$ & $18(32.1 \%)$ & $26(81.3 \%)$ & $9(75.0 \%)$ \\
\hline Hand & $63(63.0 \%)$ & $28(50.0 \%)$ & 25 (78.1\%) & $10(83.3 \%)$ \\
\hline Poliosis, n (\%) & $24(24.0 \%)$ & $16(28.6 \%)$ & $5(15.6 \%)$ & $3(25.0 \%)$ \\
\hline Active disease & 44 (44.0\%) & 24 (42.9\%) & 14 (43.8\%) & $6(50.0 \%)$ \\
\hline Confetti depigmentation & $5(5.0 \%)$ & $4(7.1 \%)$ & I (3.I\%) & $0(0.0 \%)$ \\
\hline Trichome lesion & I (I.0\%) & $0(0.0 \%)$ & I (3.I\%) & $0(0.0 \%)$ \\
\hline Köebner phenomenon & $\mathrm{I}(\mathrm{I} .0 \%)$ & $0(0.0 \%)$ & I (3.I\%) & $0(0.0 \%)$ \\
\hline Inflammatory vitiligo & $0(0.0 \%)$ & $0(0.0 \%)$ & $0(0.0 \%)$ & $0(0.0 \%)$ \\
\hline New lesion within 3 months & $41(41.0 \%)$ & 21 (37.5\%) & 14 (43.8\%) & $6(50.0 \%)$ \\
\hline Associated diseases & 14 (I4.0\%) & 8 (14.3\%) & $6(18.8 \%)$ & $0(0.0 \%)$ \\
\hline Family history of vitiligo & $24(24.0 \%)$ & II (19.6\%) & $9(28.1 \%)$ & $4(33.3 \%)$ \\
\hline
\end{tabular}

Abbreviations: BSA, body surface area; IQR, interquartile range; VES, Vitiligo Extent Score.

$p<0.001)$. The correlations between the VES, VASI and SA-VES in the $1-9.99 \%$ BSA group were higher than those in the $<1 \%$ BSA group, while the correlations in patients with $>10 \%$ BSA of vitiligo were excellent among all combinations of scoring methods (Table 3 ).
Regarding type of vitiligo, non-segmental type showed very strong and strong correlations among the VES, VASI, and SA-VES. Segmental-type vitiligo had strong correlation between the VES and VASI $(r=0.831, p<0.001)$, but only moderate correlations between the VES and SA-VES, 
Table 2 Inter-Rater Correlation of VES and VASI According to Lesion Location, BSA\%, Type of Vitiligo, and Disease Status

\begin{tabular}{|c|c|c|c|c|}
\hline & \multicolumn{2}{|c|}{ VES } & \multicolumn{2}{|c|}{ VASI } \\
\hline & ICC (95\% CI) & $\mathrm{SDC}_{95}$ & ICC (95\% CI) & $\mathrm{SDC}_{95}$ \\
\hline \multicolumn{5}{|c|}{ Location of lesions } \\
\hline All locations & $0.997(0.995-0.998)$ & 2.71 & $0.988(0.982-0.992)$ & 5.18 \\
\hline Face & $0.887(0.837-0.923)$ & 0.81 & - & - \\
\hline Trunk & $0.990(0.986-0.994)$ & 2.89 & - & - \\
\hline Extremities & $0.988(0.982-0.992)$ & 2.49 & - & - \\
\hline Feet & $0.913(0.873-0.94 I)$ & 0.94 & - & - \\
\hline Hands & $0.910(0.868-0.938)$ & 0.43 & - & - \\
\hline \multicolumn{5}{|l|}{ BSA\% } \\
\hline$<1 \%$ & $0.848(0.754-0.908)$ & 0.62 & $0.820(0.7 \mathrm{II}-0.890)$ & 0.78 \\
\hline I-9.99\% & $0.96 \mathrm{I}(0.922-0.98 \mathrm{I})$ & 3.19 & $0.932(0.865-0.966)$ & 4.34 \\
\hline$\geq 10 \%$ & $0.995(0.98 I-0.998)$ & 5.95 & $0.971(0.903-0.992)$ & 13.03 \\
\hline \multicolumn{5}{|l|}{ Type of vitiligo } \\
\hline Segmental & $0.992(0.976-0.997)$ & 1.39 & $0.985(0.957-0.995)$ & 2.01 \\
\hline Non-segmental & $0.997(0.995-0.998)$ & 2.96 & $0.988(0.98 I-0.992)$ & 5.65 \\
\hline Focal & $0.772(0.106-0.974)$ & 0.55 & $0.742(0.174-0.970)$ & 0.48 \\
\hline \multicolumn{5}{|l|}{ Disease status } \\
\hline Stable & $0.996(0.993-0.997)$ & 2.60 & $0.994(0.989-0.996)$ & 2.73 \\
\hline Active & $0.997(0.995-0.999)$ & 3.23 & $0.986(0.974-0.992)$ & 7.04 \\
\hline
\end{tabular}

Abbreviations: BSA, body surface area; Cl, confidence interval; ICC, intraclass correlation coefficient; SDC, smallest detectable change; VASI, Vitiligo Area Scoring Index; VES, Vitiligo Extent Score.

and between the VASI and SA-VES $(r=0.671,0.649$, respectively; $p<0.001)$. Focal type did not show correlations between the physicians and patients scoring methods (Table 3). Disease status did not affect the correlations among the combinations of the VES, VASI, and SA-VES scoring methods.

\section{Feasibility}

The time needed for patients to complete the SA-VES form ranged from 7.56 seconds to 3.93 minutes. The median completion time was 1.23 minutes (IQR: 0.811.73). Regarding patient rating of the difficulty of completing the SA-VES, $41 \%$ of patients rated the form as easy, followed by very easy (29\%), and moderately easy $(25 \%)$. Four patients reported the SA-VES form to be difficult, and one patient reported it to be very difficult. Some patients rated the SA-VES as difficult as a result of their having insufficient computer skills.

\section{Discussion}

Previous studies reported high reliability and validity of the VES and SA-VES. ${ }^{7,8}$ The present validation study enrolled Thai vitiligo patients of whom most had skin type IV, and we included all types of vitiligo. Our results support those from previous study that VES has very high reliability in patients with higher skin type. Moreover, reliability was good or excellent at different body locations, such as the face, trunk, extremities, hands, and feet. Previous study reported that VES is not an appropriate tool for assessing segmental vitiligo. ${ }^{8}$ In contrast, we found the VES to be a reliable scoring instrument for assessing both the affected area of vitiligo and the type of vitiligo. The ICCs of the VES in patients with $<1 \%$ BSA of vitiligo and in patients with segmental-type vitiligo were good and excellent, respectively. The VES performed overall significantly better compared to VASI for inter-rater reliability as illustrated by the non-overlapping confidence intervals. VASI seems to have more discrepancy between 2 raters than VES in patients with extensive vitiligo lesions.

Concerning validity, the correlation between physician scoring methods (VES and VASI) in this study was excellent, even in vitiligo patients with small area of lesion or segmental-type vitiligo. High correlations between the VES and SA-VES were found for each body area. We found that the face had the lowest correlation, which is similar to previous report. ${ }^{8}$ Overall correlations between physician scoring methods (VES, VASI) and patientreported outcome measurement (SA-VES) were high as 

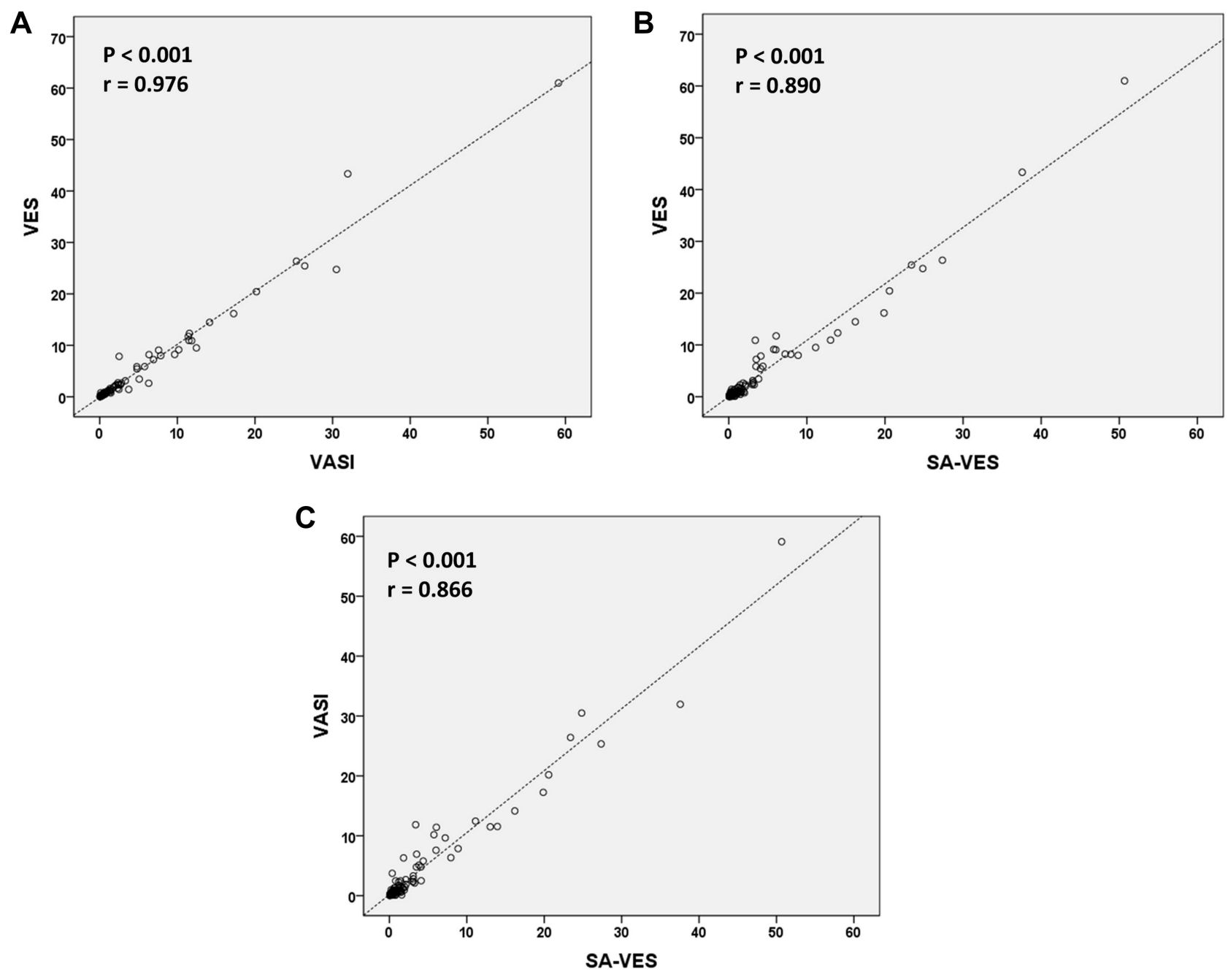

Figure I Correlation analysis between (A) the VASI and VES, (B) the VES and SA-VES, and (C) the VASI and SA-VES.

well. The results showed that the SA-VES had better correlation with the VES than with the VASI. Although overall correlation between physician and patient scoring methods was high, only moderate correlations were observed between the SA-VES and physician scoring methods (VES and VASI) in patients with vitiligo lesion $<1 \% \mathrm{BSA}$, and in patients with segmental-type vitiligo. These results were as expected since the SA-VES form has less images and less body areas than the VES. Regarding body areas, the SA-VES form does not show separation of the upper and lower face, left arm-right arm, hands, feet, or posterior area of neck. These omissions on the SA-VES could lead to confusion and inaccurate scoring in patients with a small area of vitiligo at those areas. Accordingly, this patient-reported scoring method is appropriate for use in patients with non-segmental vitiligo, and in patients with vitiligo lesion $>1 \%$ BSA.
The correlation between the VES and VASI in patients with focal-type vitiligo was excellent, but no correlation was observed between physician scoring methods and the SAVES. This result could have been influenced by errors in self-assessment made of three patients. Two patients had only one small vitiligo lesion at their forehead and right arm, respectively. Both of those patients overestimated their vitiligo severity because there was no image to select for only forehead or only right arm in the SA-VES form. In contrast, the third patient under-reported his clinical score. Unfortunately, only 15 and 5 patients with segmental and focal-type vitiligo were enrolled in this study. More participants are needed to confirm these results.

Another concern about the use of the VES and SA-VES relates to follicular repigmentation of vitiligo lesion that is not represented in either of these two instruments. This could be a cause of confusion among patients that use the SA-VES, and 

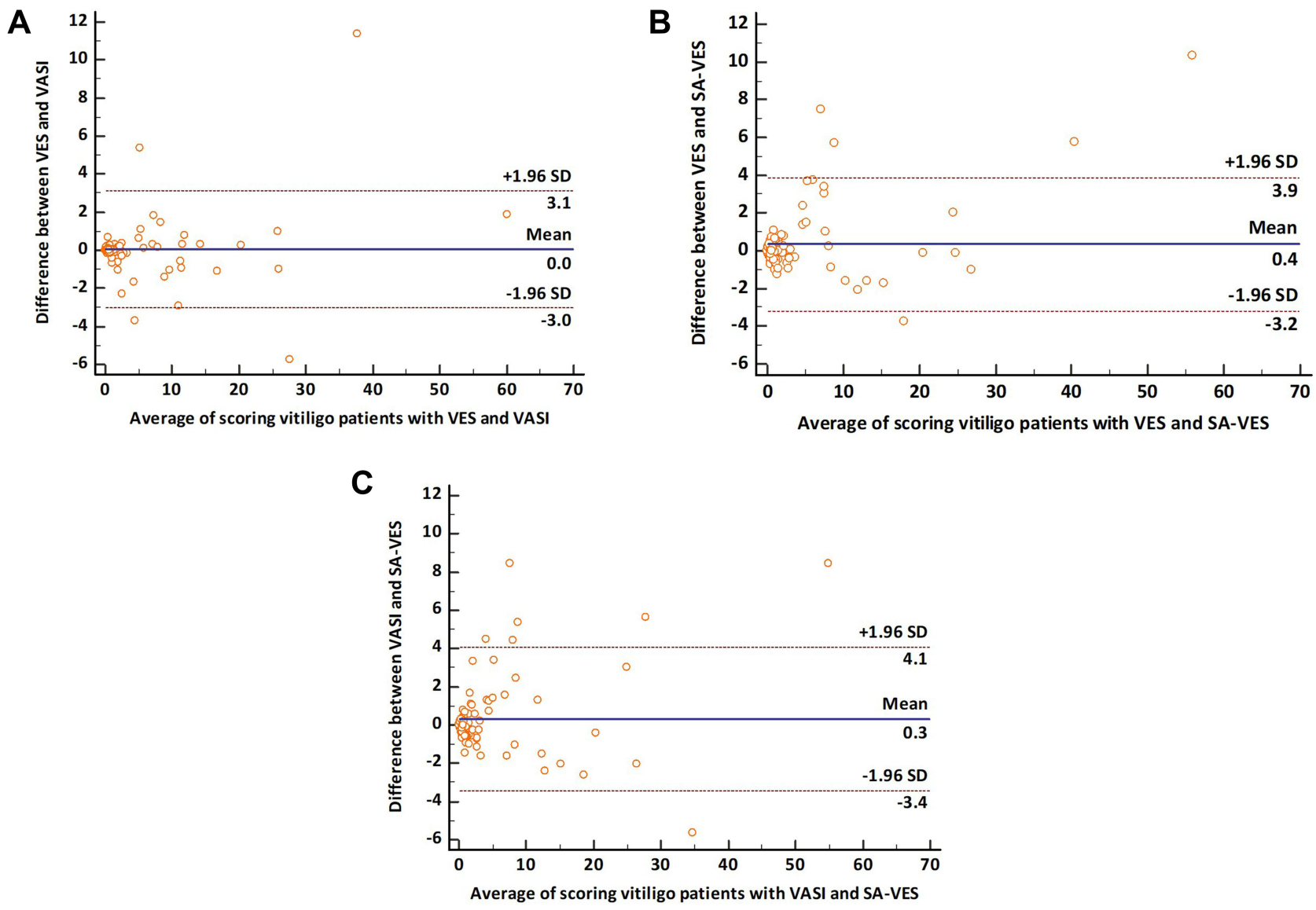

Figure 2 Bland-Altman analyses for agreement between (A) the VES and VASI, (B) the VES and SA-VES, and (C) the VASI and SA-VES.

they may be inclined to overestimate their vitiligo severity. In this study, the median SA-VES score was a little higher than the median VES and VASI scores. Our results showed good correlation between the SA-VES and the two physician scoring methods, but those correlations were both lower than the correlation between the VES and the VASI. Overestimation due to insufficient understanding of repigmentation may help to explain these results. For clinicians, van Geel et al developed the Vitiligo Extent Score-plus (VESplus) that added the perifollicular repigmentation pattern to the VES to optimize precision in severity assessment and treatment evaluation. ${ }^{11}$ The VESplus was validated and showed high correlation with the VES; however, there was no comparison with the VASI score. $^{11}$

Regarding feasibility, the advantages and strengths of the VES and SA-VES are clarity of area, user-friendliness, and intuitive use. ${ }^{7,8}$ In previous study, most patients required less than five minutes to complete the SA-VES. ${ }^{8}$ In our study, the average time to complete the SA-VES was only 1.23 minutes. Almost $70 \%$ of patients rated the difficulty of completing the SA-VES as very easy or easy, which is similar to previous study. ${ }^{8}$ Difficult or very difficult was reported in 5 patients. Since the SA-VES is a computer-based form, computer skills or academic level could be expected to influence the difficulty of completing the SA-VES form. However, the SA-VES form can also be scored on paper and the results can be calculated with a conversion table. Another patient self-assessment for scoring vitiligo is the Self-Assessment Vitiligo Area Scoring Index (SAVASI), which was successfully validated against the VASI score by Komen et al. ${ }^{12}$ These patient self-assessment scores give physicians insight into the patient's perception of their disease status, and this information may help physicians improve patient adherence to treatment.

Eighty-eight percent of patients in this study has vitiligo lesion less than $10 \%$ BSA, and with more than half (56\%) of all patients had lesion less than $1 \%$ BSA. These high proportions of low \%BSA may reflect patient concern about the disease, and an early attempt to seek treatment and control the disease. Previous study found the mean Dermatology Life Quality Index (DLQI) in Thai vitiligo 
Table 3 Spearman Correlation Analysis Between Different Instrument Combinations Relative to Location of Lesions, BSA\%, Type of Vitiligo, and Disease Stability

\begin{tabular}{|c|c|c|c|c|c|c|}
\hline & \multicolumn{2}{|c|}{ VES vs VASI } & \multicolumn{2}{|c|}{ VASI vS SA-VES } & \multicolumn{2}{|c|}{ VES vs SA-VES } \\
\hline & $r$ & $p$-value & $r$ & $p$-value & $r$ & $p$-value \\
\hline \multicolumn{7}{|c|}{ Location of lesions } \\
\hline All locations & $0.976(0.964-0.984)$ & $<0.001$ & $0.866(0.807-0.908)$ & $<0.001$ & $0.890(0.840-0.925)$ & $<0.001$ \\
\hline Face & - & - & - & - & $0.587(0.44 I-0.702)$ & $<0.001$ \\
\hline Trunk & - & - & - & - & $0.919(0.822-0.945)$ & $<0.001$ \\
\hline Extremities & - & - & - & - & $0.858(0.795-0.902)$ & $<0.001$ \\
\hline Feet & - & - & - & - & $0.907(0.865-0.937)$ & $<0.001$ \\
\hline Hands & - & - & - & - & $0.872(0.8|5-0.9| 2)$ & $<0.001$ \\
\hline \multicolumn{7}{|l|}{ BSA\% } \\
\hline$<1 \%$ & $0.892(0.822-0.936)$ & $<0.001$ & $0.537(0.319-0.701)$ & $<0.001$ & $0.584(0.379-0.734)$ & $<0.001$ \\
\hline I-9.99\% & $0.898(0.800-0.949)$ & $<0.001$ & $0.790(0.610-0.893)$ & $<0.001$ & $0.942(0.884-0.972)$ & $<0.001$ \\
\hline$\geq 10 \%$ & $0.923(0.743-0.979)$ & $<0.001$ & $0.937(0.786-0.983)$ & $<0.001$ & $0.986(0.949-0.996)$ & $<0.001$ \\
\hline \multicolumn{7}{|l|}{ Type of vitiligo } \\
\hline Segmental & 0.83 I $(0.555-0.942)$ & $<0.001$ & $0.649(0.204-0.87 I)$ & $<0.001$ & $0.671(0.124-0.850)$ & $<0.001$ \\
\hline Non-segmental & $0.970(0.953-0.980)$ & $<0.001$ & $0.846(0.769-0.899)$ & $<0.001$ & $0.883(0.823-0.924)$ & $<0.001$ \\
\hline Focal & $0.900(0.086-0.993)$ & 0.037 & $0.205(-0.827-0.921)$ & 0.741 & $0.051(-0.893-0.870)$ & 0.935 \\
\hline \multicolumn{7}{|l|}{ Disease status } \\
\hline Stable & $0.977(0.962-0.987)$ & $<0.001$ & $0.869(0.786-0.922)$ & $<0.001$ & $0.865(0.779-0.919)$ & $<0.001$ \\
\hline Active & $0.958(0.924-0.977)$ & $<0.001$ & $0.826(0.701-0.902)$ & $<0.001$ & $0.885(0.798-0.936)$ & $<0.001$ \\
\hline
\end{tabular}

Note: A p-value $<0.05$ indicates statistical significance.

Abbreviations: BSA, body surface area; SA-VES, Self-Assessment Vitiligo Extent Score; VASI, Vitiligo Area Scoring Index; VES, Vitiligo Extent Score.

patient to be $7.46 .{ }^{13}$ The prevalence of depression in Thai vitiligo patients based on the Physician Health Questionnaire (PHQ-9) was $13.5 \%$. There was moderate correlation between the DLQI score and the PHQ-9 score. Any size of vitiligo lesions tends to adversely affect patient mood and quality of life. ${ }^{13}$

This study has some mentionable limitations. First, our data was collected from a single center and there were small number of raters in the study. Second, intra-rater correlation between VES and SA-VES was not addressed. Third, half of patients in this study had less than $1 \%$ BSA involvement therefore might not represent all vitiligo patients. Lastly, there were small number of patients with segmental- and focal-type vitiligo enrolled in this study. Consequently, the results relating to segmental- and focaltype vitiligo should be interpreted with this limitation in mind.

\section{Conclusion}

In this study, we set forth to validate the VES and SAVES in Thai vitiligo population. The VES was shown to have excellent correlation compare to the VASI. The SA-
VES is a feasible and user-friendly instrument that correlated well with physicians' scoring in patients with nonsegmental or $>1 \%$ BSA vitiligo. This study showed the VES to be a reliable tool for further clinical research, as well as in routine clinical practice for assessing vitiligo severity.

\section{IRB Approval Status}

This study was reviewed and approved by the Siriraj Institutional Review Board (SIRB) (approval no. Si 512/ 2019) which was conducted in accordance with the Declaration of Helsinki.

\section{Acknowledgments}

The authors gratefully acknowledge Dr. Saowalak Hunnangkul of the Research Group and Research Network Division, Division of Clinical Epidemiology, Faculty of Medicine Siriraj Hospital, Mahidol University for assistance with statistical analysis. The authors would also like to thank the patients who generously agreed to participate in this study. 


\section{Funding}

This was an unfunded study.

\section{Disclosure}

All authors declare no personal or professional conflicts of interest relating to any aspect of this study.

\section{References}

1. Bergqvist C, Ezzedine K. Vitiligo: a review. Dermatology. 2020;236:571-592. doi:10.1159/000506103

2. Krüger C, Schallreuter KU. A review of the worldwide prevalence of vitiligo in children/adolescents and adults. Int $J$ Dermatol. 2012;51:1206-1212. doi:10.1111/j.1365-4632.2011.05377.x

3. Begum R, Marfatia YS, Laddha NC, Dwivedi M, Mansuri MS, Singh M. Vitiligo: a complex disease and a complex approach. Mol Cytogenet. 2014;7:I57. doi:10.1186/1755-8166-7-S1-I57

4. Hamzavi I, Jain H, McLean D, Shapiro J, Zeng H, Lui H. Parametric modeling of narrowband UV-B phototherapy for vitiligo using a novel quantitative tool: the Vitiligo Area Scoring Index. Arch Dermatol. 2004;140:677-683. doi:10.1001/archderm.140.6.677

5. Kohli I, Veenstra J, Hamzavi I. Vitiligo assessment methods - Vitiligo Area Scoring Index and Vitiligo European Task Force assessment. $\mathrm{Br}$ J Dermatol. 2015;172:318-319. doi:10.1111/bjd.13531

6. Alghamdi KM, Kumar A, Taïeb A, Ezzedine K. Assessment methods for the evaluation of vitiligo. J Eur Acad Dermatol Venereol. 2012;26:1463-1471.
7. van Geel N, Lommerts J, Bekkenk M, et al. Development and validation of the Vitiligo Extent Score (VES): an International Collaborative Initiative. J Invest Dermatol. 2016;136:978-984. doi:10.1016/j.jid.2015.12.040

8. van Geel N, Lommerts JE, Bekkenk MW, et al. Development and validation of a patient-reported outcome measure in vitiligo: the Self Assessment Vitiligo Extent Score (SA-VES). J Am Acad Dermatol. 2017;76:464-471. doi:10.1016/j.jaad.2016.09.034

9. Koo TK, Li MY. A guideline of selecting and reporting intraclass correlation coefficients for reliability research. J Chiropr Med. 2016;15:155-163. doi:10.1016/j.jcm.2016.02.012

10. Schober P, Boer C, Schwarte LA. Correlation coefficients: appropriate use and interpretation. Anesth Analg. 2018;126:1763-1768. doi:10.1213/ANE.0000000000002864

11. van Geel N, Bekkenk M, Lommerts JE, et al. The Vitiligo Extent Score (VES) and the VESplus are responsive instruments to assess global and regional treatment response in patients with vitiligo. $\mathrm{J} \mathrm{Am}$ Acad Dermatol. 2018;79:369-371. doi:10.1016/j.jaad.2017.12.070

12. Komen L, van der Kraaij GE, van der Veen JP, de Rie MA, Wolkerstorfer A. The validity, reliability and acceptability of the SAVASI; a new self-assessment score in vitiligo. $J$ Eur Acad Dermatol Venereol. 2015;29:2145-2151. doi:10.1111/jdv.13161

13. Silpa-Archa N, Pruksaeakanan C, Angkoolpakdeekul N, et al. Relationship between depression and quality of life among Vitiligo patients: a Self-assessment Questionnaire-based Study. Clin Cosmet Investig Dermatol. 2020;13:511-520. doi:10.2147/CCID.S265349

\section{Publish your work in this journal}

Clinical, Cosmetic and Investigational Dermatology is an international, peer-reviewed, open access, online journal that focuses on the latest clinical and experimental research in all aspects of skin disease and cosmetic interventions. This journal is indexed on CAS.
The manuscript management system is completely online and includes a very quick and fair peer-review system, which is all easy to use. Visit http://www.dovepress.com/testimonials.php to read real quotes from published authors. 